\title{
The Life Prediction Model of Vehicles Engine Based on Cylinder Wear-law
}

\author{
TANG Yanfeng ${ }^{1, a}$, FENG Huijuan ${ }^{1, b}$, ZHANG Jian $^{1, c}$ \\ ${ }^{1}$ Department of Automobile Engineering, Military Transportation University, Tianjin 300161, China \\ ajjxy_tang@163.com, bbluebellfhj@163.com, yitzhak@163.com
}

Keywords: Vehicles Engine, Life Prediction, Statistical Distribution Model, Wear-Law

\begin{abstract}
The wear-law and the life prediction model of mechanical components were analyzed. Supposing the mean wearing rate of cylinder during the stable wear period obeys normal distribution, the samples of cylinder life was acquired applying the time-step Monte Carlo simulation method, and the life distribution function of cylinder wear was defined applying Kolmoglov hypothesis test. Finally, the life prediction model of vehicles engine was build based on cylinder wear-law. The example proves that the model build here can predict the life of gradual changed components, and it well compliant to predict the life of vehicles engine.
\end{abstract}

\section{Introduction}

Wear is the main destroy form of mechanical components. Relative motions exist in many mechanical components, and the friction, wear and lubrication between the kinematic pair produce direct impact to machinery's performance such as function, efficiency, reliability and life ${ }^{[1]}$. Predicting the life of mechanical components by rule and line has practical significance for drafting maintenance plan. Along with the deep-going of tribology research and the development of reliability engineering, building the reliability model for wear and then predicting life of mechanical components become an common method, which can by researching the friction-mechanism and wear-law of mechanical components. Now, there are two methods to predict the life of mechanical systems. One is based on theoretical model of tribology, which can predict the life according to the wear-law, and the other is based on statistical model of wear measurement, which can predict the life according to statistics law.

There has the same condition between the friction pair of vehicles engine cylinder and the friction of other mechanical systems, that the normal wear process can be divided into three periods ${ }^{[2]}$. It usually thinks that the work time in the stable wear period is its life, and the wearing capacity here is the limit wearing capacity. Just because there exists a stable linear wear area and the wearing rate in this area is approximate a constant in wear of the mechanical components, so the life of the mechanical components can be predicted through this area.

Now, there are many statistical models to predict the life of the mechanical components, which can sum up to three types as follows:

(1) The method of linear estimation. Supposing the wearing rate is a fixed value in the stable wear period, the theoretical life of the components can be estimated by its limit wearing capacity, its actual wearing capacity and its accumulated running time when measured.

(2) The reliability model when the wear capacity obeys normal distribution ${ }^{[3-5]}$.

(3) The reliability model based on life distribution ${ }^{[6]}$.

Among the three models above, the merit of the first model is relatively simple, and suit to estimate the life of single component, but can not reflect the randomness in the wearing. The second model and the third model can support the work of life prediction, parameter estimation and reliability modeling, but they further suit to deal with the test data obtained in strict conditions but not the scattered data, just because they based on the suppose of normal distribution.

However, the wear data maybe scattered in general, and the life statistical data is difficult to be obtained. Thus, the distribution of wearing rate is adopted to predict the life. 


\section{Process of Life Prediction Based on Statistical Method}

\section{Define the Wearing Rate and Its Distribution}

There are $n$ vehicles in the experiment which limited to stable wear period. The $i$ cylinder of one of the vehicles begins work at $T_{B i}$ with the wearing capacity $w_{o i}$ and ends work at $T_{E i}$ with the wearing capacity $w_{i}$. Thus, the average wearing rate $x_{i}$ of the cylinder'can be calculated by

$$
x_{i}=\frac{w_{i}-w_{0 i}}{T_{E i}-T_{B i}}
$$

If the begin time or the wearing capacity beginning is unknown, the average wearing rate can be estimated by

$$
x_{i}=\frac{w_{i}}{T_{E i}}
$$

The average wearing rate which has randomness is different for different cylinders because of the different factors as machining accuracy or assembly quality. Thus, the average wearing rate of cylinder can be described by a random variable $X$, and the average wearing rate of $n$ cylinders obtained in the experiment can be a simple random sample of $X$, that is

$$
X=\left\{x_{1}, x_{2}, x_{3}, \ldots, x_{i}, \ldots, x_{n}\right\}
$$

Supposing $X$ obeys normal distribution, its mean value and variance can be estimated by

$$
\bar{X}=\frac{1}{n} \sum_{i=1}^{n} x_{i} \quad S^{2}=\frac{1}{n-1} \sum_{i=1}^{n}\left(x_{i}-\bar{X}\right)^{2}
$$

The distribution function of its average wearing rate is

$$
F(x)=\int_{0}^{x} \frac{1}{s \sqrt{2 \pi}} e^{-\frac{(t-\bar{X})^{2}}{2 s^{2}}} d t
$$

\section{Service Life Simulation}

Applying the time-step Monte Carlo simulation method, the simulation is started by choosing the proper step and using the engine's operating time as simulation clock. Draw the samples of wearing rate distribution randomly, and estimate the total wearing capacity after every sampling, until this simulation ends when the limit wearing capacity is achieved. That is, if the wearing capacity is less than the limit wearing capacity, continues to draw the samples randomly until the wearing capacity is achieved or exceeded the limit wearing capacity, at which time the next simulation can be started. Simulating $n$ times, $n$ sample values of the cylinder's life are obtained. The reliability model of the cylinder can be obtained through analyzing the simulation results by statistic method.

Setting up the simulation times as $N$, the limit wearing capacity as $W_{P}$, the simulation beginning time as $T_{\mathrm{B}}$, then the sample value of the cylinder's life obtained in the simulation $i$ is

$$
T=\left(T_{1}, T_{2}, T_{3}, \ldots, T_{i}, \ldots, T_{N}\right)
$$

Recording the running state in simulation $i$, including the now simulation times, the total wearing capacity and the total running time and so on. It can go to the step five when the simulation times $(i>N-1)$ are achieved.

The sampling formula of wearing rate is

$$
x=S \times \sqrt{-2 \ln r_{1}} \sin \left(2 \pi r_{2}\right)+\bar{X}
$$

or

$$
x=S \times \sqrt{-2 \ln r_{1}} \cos \left(2 \pi r_{2}\right)+\bar{X}
$$

In above formula, $r_{1}$ and $r_{2}$ are the independent random numbers distributing uniformly at the interval $(0,1)$.

Then the total wearing capacity in sampling $i$ is

$$
W\left(t_{i}\right)=W\left(t_{i-1}\right)+\left(t_{i}-t_{i-1}\right) x_{i}
$$

In above formula, $t_{i}$ is the total working time of the cylinder at the sampling point $i(\mathrm{i}=0,1,2,3 \ldots)$, and it is $T_{A}$ when $x_{i}$ is the sampling wearing rate of cylinder at $t_{i}$; $W\left(t_{i}\right)$ is the total wearing capacity of cylinder at $t_{i}, \mathrm{i}=0,1,2,3 \ldots$, and it is $W_{A}$ when $i=0$. 
When $W\left(t_{i}\right)=W_{P}$, the $T_{i}=t_{i}$, then go to step two, and start the next simulation.

When $W\left(t_{i}\right)<W_{P}$, the $T_{i}=t_{i}$, then go to step three.

When $W\left(t_{i}\right)>W_{P}$, the $T_{i}=t_{i}-\frac{W\left(t_{i}\right)-W_{P}}{x_{i}}$, then go to step two, and start the next simulation.

Base on four distribution types frequently-used in reliability engineering, which are exponential distribution, normal distribution, logarithmic normal distribution and Weibull distribution, the Kolmoglov hypothesis test is applied to define the life distribution form and the distribution parameters. Its fundamental is:

Setting up $x_{1}, x_{2}, \cdots x_{n}$ is the sample observation taking from the distribution function $F(x)$, test the hypothesis $H_{0}: F(x)=F_{0}(x)$, and the backup hypothesis $H_{1}: F(x) \neq F_{0}(x)$.

Testing steps:

(1) If hypothesis $H_{0}: F(x)=F_{0}(x)$ is established, then the backup hypothesis $H_{1}: F(x) \neq F_{0}(x)$.

(2) Define the statistic and its distribution used in testing. If hypothesis $H_{0}: F(x)=F_{0}(x)$ is established, $F_{0}(x)$ is the sample distribution function, and then the statistic $D_{n}=\underset{-\infty<x<+\infty}{S U P}\left|F(i)-F_{0}(x)\right|$ will not too big. But if the hypothesis is not established, the statistic has the trends of turning bigger. $F(i)=\frac{i}{n+1}$ is the per cent depot to calculate the failure interval $i$.

(3) Given the significance $\alpha$ to seek rejection regions. Set up $P\left\{D_{n}>D_{n, \alpha}\right\}=\alpha$, and the rejection regions is $D_{n}>D_{n, \alpha}$. If $\alpha$ is given, $D_{n, \alpha}$ can traced by $K-S$ check list.

(4) According to the sample observation and the given $F_{0}(x)$, the statistic $D_{n}$ is

$D_{n}=S_{-\infty<x<+\infty}\left|F(i)-F_{0}(x)\right|=\max \left\{\left|F(i)-F_{0}\left(x_{i}\right)\right|\right\} \quad i=1,2, \cdots n$

(5) According to above alqorithm, four statistic $D_{n}$ are obtained for the four distribution types in reliability engineering, and the distribution type corresponding to the minimum $D_{n}$ is the optimal.

\section{Example}

It is the test wear data of 9 vehicles cylinder in a certain statistic experiment.

Table1 the testing wear data of engine cylinder

\begin{tabular}{|c|c|c|c|c|c|}
\hline No. & $\begin{array}{c}\text { serial } \\
\text { number }\end{array}$ & $\begin{array}{c}\text { Start mileage } \\
(\mathrm{km})\end{array}$ & $\begin{array}{c}\text { Testing mileage } \\
(\mathrm{km})\end{array}$ & $\begin{array}{c}\text { Maximum } \\
\text { wearing capacity } \\
(\mathrm{mm})\end{array}$ & $\begin{array}{c}\text { Average } \\
\text { wearing rate } \\
\left(\mathrm{mm} / 10^{4} \mathrm{~km}\right)\end{array}$ \\
\hline 1 & $1 \#$ & 9874 & 59967 & 0.015 & 0.002501 \\
\hline 2 & $2 \#$ & 19503 & 69504 & 0.020 & 0.002878 \\
\hline 3 & $3 \#$ & 94857 & 144713 & 0.045 & 0.003110 \\
\hline 4 & $4 \#$ & 427 & 49800 & 0.020 & 0.004016 \\
\hline 5 & $5 \#$ & 38648 & 87161 & 0.050 & 0.005737 \\
\hline 6 & $6 \#$ & 71366 & 119255 & 0.050 & 0.004193 \\
\hline 7 & $7 \#$ & 617 & 50186 & 0.040 & 0.007970 \\
\hline 8 & $8 \#$ & 68561 & 119336 & 0.050 & 0.004190 \\
\hline 9 & $9 \#$ & 68930 & 118835 & 0.030 & 0.002525 \\
\hline
\end{tabular}

According to the formula (4), the mean value and the mean square deviation of average wearing rate are $0.004-124$ and $0.0017-745$.

Take the step as $1000 \mathrm{~km}$ and the limit wearing capacity as $0.050 \mathrm{~mm}$, simulating 1000 times, the 1000 life samples are obtained as $(121447.73,110917.57,114273.93,115593.24,134580.68, \ldots$, 119670.63, 117508.39, 117884.53, 131045.45, 118942.32). 
Through the hypothesis test of the life sample, the life distribution obtained obeys normal distribution, and its mean value and mean square deviation are 121009.18 and 4453.51.The distribution density function is

$$
f(t)=\frac{1}{\sqrt{2 \pi} 4453.51} \exp \left[-\frac{1}{2}\left(\frac{t-121009.18}{4453.51}\right)^{2}\right]
$$

The life-span characteristics is as the Table 2.

Table2 the life-span characteristics of samples $(\mathrm{km})$

\begin{tabular}{|c|c|c|}
\hline Order Number & Parameter & Value \\
\hline 1 & Average life & 121009 \\
\hline 2 & Life variance & 4454 \\
\hline 3 & Median life & 121009 \\
\hline 4 & Characteristics life & 122107 \\
\hline 5 & Reliabilitylife & 113706 \\
\hline
\end{tabular}

The average life of this engine is $121009 \mathrm{~km}$, which has better uniformity than the estimated result(121 $233 \mathrm{~km}$ ) in model (1). In order to ensure the higher reliability of the engine, the repair can be take at the mileage of $113706 \mathrm{~km}$.

\section{Conclusions}

A method based on statistical distribution model is discussed in this paper to predict the life of the vehicles engine. It shows that, it can predict the life of gradual changed components commendably applying the statistical distribution model in this paper, and suit to deal with the data of wear test and locale statistic. It also shows that, when applying the statistic distribution model, it is more accuracy when the sample data is bigger. It can be popularized to other machinery parts has the same wear-law, such as engine crankshaft, transmission gear and so on.

\section{References}

[1] WANG Chengtao. Vehicle Tribology. Shanghai Jiao Tong UniversityPress,2002, p. 2

[2] ZHANG Dong. Falut Analysis. National Defense Industry Press,2004, p. 229-230

[3] Kececioglu, D. B.\&Feng-Bin Sun. Wear reliability and preventive replacement policy for mechanical components. //The 47th meeting of Mechanical Failure Prevent Group, 1993, p.263-278. [4] Liu Xiangkai.Yuan Hongtao.Gan Maozhi.Shi Quan.Research on a Reliability Calculating Model Based on Wear Data. //IJPEM,2000, p.5.

[5] Liu Xiangkai.Li lianjun.Gan Maozhi.Yuan Hongtao.Research and Accomplishment of the Analytic Model of Products' Wear Laws. //Proceedings of the 7th International Conference on IE\&EM,. 2000, p.28-30.

[6] MIL-HDBK-338B, ELECTRONIC RELIABILITY DESIGN HANDBOOK,1,OCT 1998, p.5-7. 